\title{
Coincidental CMV Colitis Post-hemorrhoidopexy in an Immunocompetent patient
}

\author{
Ajda Altinoz ${ }^{1 *}$, Huda Gasmelseed ${ }^{2}$, Katharina Kessler ${ }^{3}$ \\ ${ }^{1}$ Division of General Surgery, Tawam Hospital, Al Ain, Abu Dhabi, United Arab Emirates, \\ ${ }^{2}$ Division of Infectious Diseases, Al Ain Hospital, Al Ain, Abu Dhabi, United Arab Emirates, \\ ${ }^{3}$ Division of General Surgery, Al Ain Hospital, Al Ain, Abu Dhabi, United Arab Emirates, \\ *Corresponding Author:Ajda Altinoz, General Surgery Resident Tawam Hospital, Al Ain,Abu Dhabi, United Arab Emirates. \\ Email: ajtinoz@seha.ae \\ Received Date: November 6, 2019; Accepted Date: November 12, 2019; Published Date: November $18,2019$. \\ Citation: Ajda Altinoz, Gasmelseed H, Kessler K. 2019) Coincidental CMV Colitis Post-hemorrhoidopexy in an Immunocompetent patient. \\ Surgical Case Reports and Images, 2(1); Doi:10.31579/JSCR/2019/008 \\ Copyright: (2019. Ajda Altinoz. This is an open-access article distributed under the terms of the Creative Commons Attribution License, \\ which permits unrestricted use, distribution, and reproduction in any medium, provided the original author and source are credited.
}

Abstract
This is a case of a 74 years old immunocompetent female who developed severe diarrhea after haemorrhoidopexy for
2 months duration. Investigations to find the cause of persistent diarrhea were done, including colonoscopy showed
ulcers from which biopsy was taken and the presence of CMV-DNA was identified. She got treated with IV and oral
Ganciclovir and repeated colonoscopy showed improvement of the ulcers with gradually improvement of diarrhea.
Keywords: Cytomegalovirus, hemorrhoidopexy, immunocompetent
Acknowlegdement - Not Applicable
Abbreviations: CMV - Cytomegalovirus

\section{Introduction}

Cytomegalovirus (CMV) colitis usually occurs in patients with advanced immunosuppression. In immunocompetent patient it is an extreme rare finding. CMV can infect the entire gastrointestinal tract. However, the colon is the most frequent site in immunocompetent patients [1]. CMV colitis clinically presents with a low-grade fever, weight loss, anorexia, malaise, and abdominal pain [2]. Furthermore, watery diarrhoea and haematochezia are other common manifestations. Mucosal haemorrhage and perforation can be designated as life-threatening complications of CMV colitis [2]. Often it is misleading as ulcerative colitis. Missed diagnoses of CMV Colitis can lead to ischemia and transmural necrosis resulting in perforation and peritonitis.

CMV is a double-stranded DNA virus belonging to the herpes virus family. The prevalence of CMV infections in the general adult population is approximately $40 \%-100 \%$ [3]. CMV infections are common worldwide due to its excretion in body fluids and transmission by close personal contact [4]. In normal hosts, primary infection is usually asymptomatic, but sometimes can result in mononucleosis-like syndrome, accompanied by symptoms such as fever, myalgia, cervical lymphadenopathy and elevated liver enzymes [4].

The first report highlighting CMV colitis in an immunocompetent patient was published in 1992. Since then, several cases have been reported in patients with chronic renal insufficiency and hemodialysis, co-infection with bacterial gastrointestinal infections, and food allergy [3-4].

Stapled hemorrhoidopexy is a safe and effective procedure for prolapsed haemorrhoids. It is associated with less postoperative pain, but higher rate of re-intervention for residual prolapse compared to conventional hemorrhoidectomy [5].

\section{Case Presentation}

A 74 years old female, with multiple comorbidities including complicated diabetes mellitus type 2, hypertension, Parkinson's disease, and cerebrovascular disease, presented with 2 months history of severe watery diarrhea occasionally associated with blood. Prior to the presentation patient underwent stapled hemorrhoidopexy for prolapsed bleeding haemorrhoids with uneventful immediate postoperative course. After 3 weeks of the operation she developed diarrhea and continued despite stopping laxatives. A colonoscopy done prior to hemorrhoidopexy was unremarkable except for a polyp. Polypectomy was performed which revealed non-malignant pathology. Frequency of diarrhoea progressed over 1 month. It was associated with abdominal cramps, bloating, nausea and anal pain.

On physical examination, patient was hemodynamically stable with pulse of $74 \mathrm{bpm}$ and blood pressure 132/86 $\mathrm{mmHg}$. Abdominal examination revealed soft and diffuse mild tenderness. No peritonitis signs. Digital rectal examination showed blood tinged watery stool. Laboratory investigations showed white blood count of $12,000 / \mathrm{mm}^{3}$, c-reactive protein(CRP) $134 \mathrm{mg} / \mathrm{L}$ and haemoglobin of $8.7 \mathrm{~g} / \mathrm{dL}$.

Admission was required for rehydration. Empiric treatment with Metronidazole as well as Mesalamine was started, but did not improve the diarrhoea. The patient developed acute blood loss anemia due to hematochezia that required blood transfusions.

A Computerized Tomogram (CT) of the abdomen and pelvis showed recto-sigmoid oedematous wall thickening and infiltration in the surrounding fat (Image 1). 


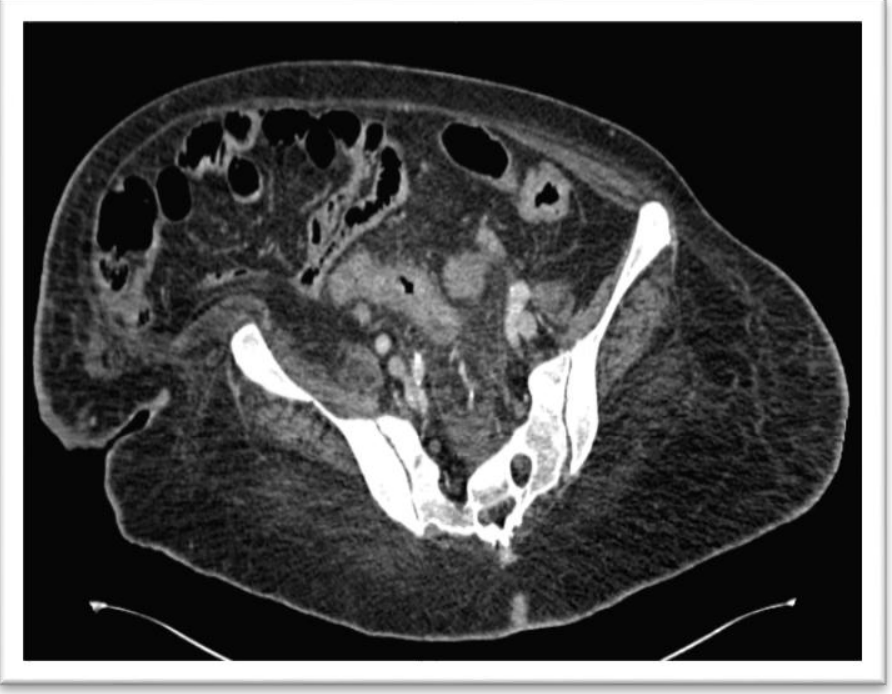

Image 1. CT abdomen showing recto-sigmoid wall thickening and surrounding fat stranding

There was thin streak of fluid in the abdomen. A colonoscopy performed revealed multiple linear and deep ulcerated lesions with white slough both in sigmoid colon and rectum up to $30 \mathrm{~cm}$ with fragile mucosa (Image 2).

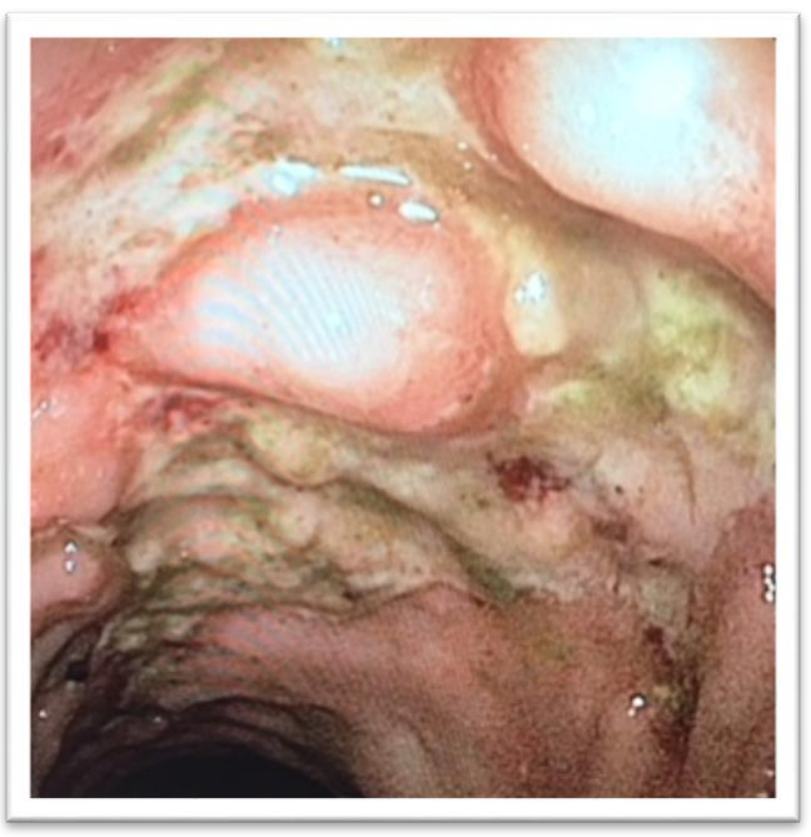

Image 2: Colonoscopy at rectosigmoid region showing ulcers with white slough

Random biopsies were taken from the entire colon and terminal ileum. All stool tests were negative. HIV testing was negative. Clostridium difficile toxin was tested 3 times and all were negative. Further testing of the biopsies revealed presence of CMV-DNA.

The patient was put on treatment with Ganciclovir intravenously (IV) for 2 weeks, followed by oral Ganciclovir for another 4 weeks to treat cytomegalovirus colitis. The condition of the patient improved slowly. Six weeks after treatment, colonoscopy repeated revealed few superficial ulcers in the rectum and previous ulcer showing signs of healing without any evidence of CMV-DNA in the biopsies. Six months after the treatment the patient still having infrequent loose stool, but no diarrhoea features and bleeding per rectum.

Auctores Publishing - Volume 2(1)-008 www.auctoresonline.org Page - 2
On follow up after 1 year of hospitalization and treatment for CMV colitis, patient has improved and diarrhoea subsided slowly.

\section{Discussion}

Recently, a rapidly rising number of literature cases worldwide indicate that CMV infections can also be observed in immunocompetent individuals. It usually results in gastroenteritis, duodenitis, ileitis, proctitis, or exacerbation of inflammatory bowel disease (IBD). However, colitis secondary to CMV infection is a rare clinical pathological entity.

Clinical as well as endoscopic features of this condition are nonspecific. Staining of colonic mucosal tissue with hematoxylin and eosin may show the typical viral inclusions associated with CMV colitis that are highly specific [6]. However, the staining has low sensitivity compared to immunohistochemistry, which is considered the gold standard for diagnosis of CMV colitis [6]. A high index of suspicion is needed, and once diagnosis is made, treatment should be highly considered to improve the outcome.

There are several agents available for the systemic therapy of CMV infection, including Ganciclovir, Valganciclovir, Foscarnet, and Cidofovir. The efficacy and toxicities of these agents have been evaluated extensively only in immunocompromised patients. However, clinical utility of these agents in the immunocompetent hosts remains unproven. Doses of the drugs and the duration of therapy also have not been clarified for this group of patients. Currently, Ganciclovir is the preferred antiviral drug for the treatment of CMV infection in immunocompetent patients. The prognosis in patients with CMV colitis can be improved markedly by both Ganciclovir and Foscarnet [7-9]. However, Ganciclovir can result in serious adverse reactions, including myelosuppression, central nervous system disorders, hepatotoxicity and nephrotoxicity [4]. Despite these serious side effects, antiviral therapy is recommended for immunocompetent patients because without antiviral treatment CMV colitis results in poor outcomes [1].

This case caused diagnostic dilemma, as only a few cases exist that highlight CMV colitis after stapled hemorrhoidopexy. Meshikhes et al, presented a case of a male that presented with bleeding per rectum associated with multiple polypoid lesions 6 months after stapled hemorrhoidopexy [10]. Differences to our case, other than clinical presentation and pathological lesions, are that our patient had a prior colonoscopy with insignificant findings before procedure. For the other case, there were no prior colonoscopy and no documented operative findings as procedure was carried out at some other centre possibility of prior CMV infection before hemorrhoidopexy exists. Nevertheless, CMV colitis is not a complication after hemorrhoidopexy, but a coincidental finding.

In conclusion, CMV colitis should be considered in immunocompetent patients, especially after exclusion of more common aetiologies for severe diarrhoea. The diagnosis of CMV colitis can be established on the basis of biopsies or Polymerase Chain Reaction tests. Timely diagnosis and treatment are essential in order to improve the outcome in elderly patients or patients with serious comorbidities.

Contributions of Authors - A Altinoz has written and revised the article. K Kessler acquired data drafted and revised part of the article. H Gasmelseed acquired data and revised the article.

Competing Interest - Authors have no declaration. No conflict of interest.

\section{References}

1. Galiatsatos P, Shrier I, Lamoureux E, Szilagyi A. Meta-analysis of outcome of cytomegalovirus colitis in immunocompetent hosts. Dig Dis Sci. 2005;50:609-616. 
2. Gorsane I, Aloui S, Letaif A, Hadhri R, Haouala F, Frih A, et al. Cytomegalovirus ischemic colitis and transverse myelitis in a renal transplant recipient. Saudi J Kidney Dis Transpl. 2013;24:309-314.

3. Nakase H, Herfarth H. Cytomegalovirus colitis, cytomegalovirus hepatitis and systemic cytomegalovirus infection: common features and differences. Inflamm Intest Dis. 2016;1:15-23.

4. Rafailidis PI, Mourtzoukou EG, Varbobitis IC, Falagas ME. Severe cytomegalovirus infection in apparently immunocompetent patients: a systematic review. Virol J. 2008;5:47.

5. Burch J, Epstein D, Baba-Akbari A, Weatherly H, Fox D, Golder S, Jayne D, Drummond M, Woolacott N. Stapled haemorrhoidectomy (haemorrhoidopexy) for the treatment of haemorrhoids: a systematic review and economic evaluation. Health Technol Assess. 2008 Apr;12(8):iii-iv, ix-x, 1-193.
6. L. Goodman, C. D. Murray, J. Watkins, P. D. Griffiths, and D. P. Webster. CMV in the gut: a critical review of CMV detection in the immunocompetent host with colitis. Eur J Clin Microbiol Infect Dis. 2015; 34(1): 13-18.

7. Chang HR, Lian JD, Chan CH, Wong LC. Cytomegalovirus ischemic colitis of a diabetic renal transplant recipient. Clin Transplant. 2004;18:100-104.

8. Hasegawa T, Aomatsu K, Nakamura M, Aomatsu N, Aomatsu K. Cytomegalovirus colitis followed by ischemic colitis in a nonimmunocompromised adult: a case report. World J Gastroenterol. 2015;21:3750-3754.

9. Termsarasab P, Katirji B. Opportunistic infections in myasthenia gravis treated with mycophenolate mofetil. J Neuroimmunol. 2012;249:83-85.

10. Meshikhes AWN and Issa H. Staple Line Polyposis and Cytomegalovirus Infection after Stapled Haemorrhoidectomy. Case Rep Gastroenterol. 2010 May-Aug; 4(2): 204-209. 